\title{
Advances in imaging
}

\author{
Andreas G. Wibmer, Hebert Alberto Vargas, Hedvig Hricak \\ Memorial Sloan Kettering Cancer Center, Department of Radiology, 1275 York Avenue, New York, \\ New York 10065, USA.
}

\section{Abstract}

In the past year, the results of three studies in the field of prostate cancer imaging — the prostate MR imaging study (PROMIS), an analysis of the cost-effectiveness of various diagnostic strategies based on PROMIS data, and a retrospective analysis of a prostate-specific membrane antigen (PSMA)-directed PET radiopharmaceutical — have been published that could have lasting effects on clinical practice.

Over the past year, the scholarly literature on imaging of prostate cancer continued to expand rapidly: since October 2016, >1,400 articles on prostate cancer imaging have been listed on the PubMed database. Results from three papers in particular could have especially strong, sustained effects on clinical practice: the prostate MR imaging study (PROMIS) ${ }^{1}$, an analysis of the cost-effectiveness of various diagnostic strategies based on PROMIS data ${ }^{2}$, and a retrospective analysis of a prostate-specific membrane antigen (PSMA, also known as glutamate carboxypeptidase 2)-directed PET radiopharmaceutical ${ }^{3}$.

For diagnosis of suspected primary prostate cancer, the exact role of prostate MRI in relation to transrectal ultrasonography- guided (TRUS) biopsy has been debated for over a decade. PROMIS, published in February 2017, was a prospective multicentre investigation directly comparing the diagnostic precision of the two tests in a cohort of 576 men with suspected prostate cancer ${ }^{1}$.

As part of the PROMIS protocol, biopsy- naive patients with elevated serum PSA, suspicious digital rectal examination, or a family history of prostate cancer underwent a standardized 1.5 T prostate MRI examination that comprised T1-weighted, T2-weighted, diffusion-weighted, and contrast-enhanced sequences. All study radiologists were specialists in urogenital imaging, underwent additional specific training before the start of the study, and were provided with the patients' clinical details. The likelihood of prostate cancer was rated on a 5-point Likert scale, ranging from 1 (highly unlikely) to 5 (highly likely), and an MRI score $\geq 3$ was regarded as positive for the presence of prostate cancer $(418 / 576,73 \%)$. With patients, clinicians, and pathologists blinded to the MRI results, patients underwent combined prostate biopsy sampling, including sequential transperineal template prostate mapping (TPM, the reference standard) and 12-core TRUS biopsy. On histopathological examination, clinically significant prostate cancer was defined as the presence of any Gleason score $\geq 4+3$ or any cancer core length $\succeq 6 \mathrm{~mm}(230 / 576,40 \%)$. In the head-to-head 
comparison, prostate MRI was more sensitive than TRUS biopsy (93\% versus $48 \%, P<$ $0.0001)$ and less specific ( $41 \%$ versus $96 \%, P<0.0001)$. These results translated to a significantly higher NPV (89\% versus $74 \%, P<0.0001)$ and significantly lower PPV (51\% versus $90 \%, P<0.0001$ ) for MRI than for TRUS biopsy. TRUS biopsy missed 119 of 230 (52\%) instances of clinically significant prostate cancer, whereas MRI missed 17 of 230 (7\%) instances. Notably, MRI identified all cases with a Gleason score $\geq 4+3$; the 17 cases missed on MRI were classified as clinically significant based on maximum cancer core length alone. The authors concluded that $\sim 25 \%$ of patients with a clinical suspicion of prostate cancer and a negative MRI could safely forgo TRUS biopsy.

A companion publication evaluated the cost-effectiveness of various diagnostic strategies based on the PROMIS data ${ }^{2}$. This study evaluated 383 possible combinations of prostate MRI, systematic TRUS biopsy, MRI-targeted TRUS biopsy, and TPM biopsy. The analysis showed that, of all combinations with initial MRI and subsequent targeted TRUS biopsies of suspicious lesions, the most cost-effective strategies would detect $85-95 \%$ of all clinically significant cancers at a cost of $£ 628-807$ per patient. For TRUS-biopsy-first strategies, the most cost-effective, in which MRI and targeted biopsies would be performed only in patients with negative initial TRUS biopsy, would detect $91 \%$ of all clinically significant cancers at a cost of $£ 709$ per patient. The authors concluded that prostate MRI followed by targeted TRUS biopsy of suspicious lesions would be cost- effective for prostate cancer diagnosis.

PROMIS is the largest prospective trial providing level 1 evidence for the value of prostate MRI as a triage test in patients with clinical suspicion of prostate cancer. The most important finding from this study is that MRI seems to provide the high sensitivity and NPV needed for it to be a clinically useful screening tool. The results of PROMIS corroborate previous reports, as summarized in a 2017 meta-analysis of 48 studies (9,613 patients), among which the median NPV for clinically significant prostate cancer was $88 \% 4$.

Reasonable arguments exist supporting and questioning the validity and applicability of the PROMIS results. The main criticism is that the high quality of MRI and expertise of radiologists involved in PROMIS would be difficult to achieve in general practice. In a realworld scenario, the sensitivity of prostate MRI would probably be lower than that reported in PROMIS. However, the prevalence of clinically significant prostate cancer in the PROMIS population (40\%) was higher than would be expected in the general population (in fact, the sample size calculations for PROMIS were based on a prevalence of $15 \%$ ). With lower disease prevalence in the tested population, the NPV of MRI would probably stay high, even with a slightly reduced sensitivity. 


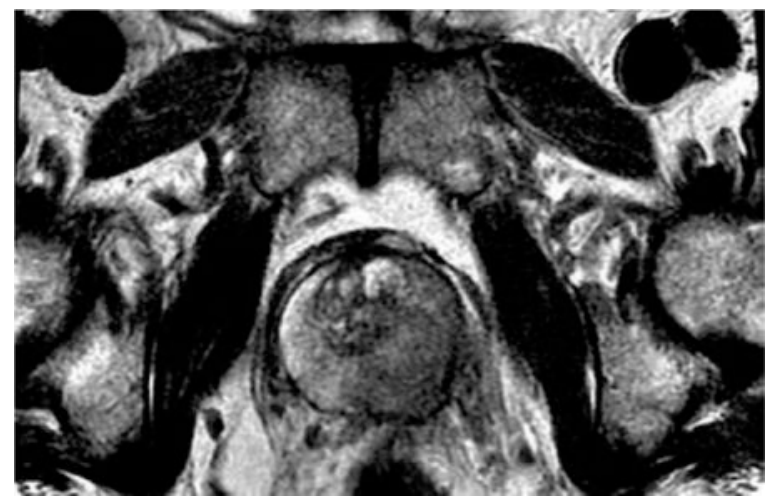

In the cost-effectiveness analysis, MRI-first strategies seem to be slightly more efficient but also more expensive than TRUS-first strategies. Caution is needed when translating these numbers into clinical practice. First, the calculations were exclusively based on the UK health-care system, which might be different from those of other regions. Second, cancer detection rates might be higher with computational MRI-ultrasonography-fusion biopsy, which is now routinely performed in many countries, than with cognitively targeted TRUS biopsy ${ }^{5}$, although some investigators have not found a significant difference between the two methods ${ }^{6}$. The efficacy of MRI-first strategies and their costs might depend, therefore, on the biopsy sampling technique used, which was not taken into account in the PROMIS costeffectiveness analysis. In addition, changing the definition of clinically significant prostate cancer on histopathology will alter the diagnostic performance of prostate MRI, as shown in PROMIS and previous reports. However, whether biopsy findings alone can truly indicate clinical significance, which is ultimately defined by the occurrence of adverse clinical events (such as the development of symptomatic disease, need for therapy, or death from cancer) is questionable. For PROMIS, study participants consented to long-term follow-up monitoring for cancer outcomes and mortality. These data will hopefully enable assessment of the true value of prostate MRI, TPM biopsy, and TRUS biopsy, not only from a diagnostic perspective but also from a prognostic one.

Regarding recurrent prostate cancer, a growing body of evidence suggests that patients with local tumour recurrence after radical treatment or oligometastatic disease might benefit from targeted therapies ${ }^{7}$. In this scenario, imaging is needed to localize disease sites. Up until a few years ago, the available conventional imaging methods, including CT, bone scintigraphy, or PET-CT with ${ }^{18}$ F-labelled glucose or sodium fluoride, were of limited sensitivity, particularly in patients with early biochemical recurrence (such as PSA levels $<1 \mathrm{ng} / \mathrm{ml}$ after prostatectomy $)^{8}$. Some PET radiopharmaceuticals recently approved by the FDA, including ${ }^{11} \mathrm{C}$-choline and ${ }^{18} \mathrm{~F}$-fluciclovine, are also of limited use in patients with such low PSA levels ${ }^{8}$. However, in 2011, PET probes targeting PSMA became available for clinical use. PSMA is highly overexpressed in prostate cancer. A retrospective analysis of a PSMAdirected PET radiopharmaceutical in a consecutive series of 1,007 patients with recurrent prostate cancer was published in May $2017^{3}$. Patients had initially been treated with surgery, radio-therapy, hormonal therapy, high-intensity focused ultrasound ablation, or a combination of these therapies with or without chemo-therapy. All patients underwent a standardized PET-CT examination 1 hour after injection of ${ }^{68}$ Ga-PSMA-11. The primary 
end point was positivity of the imaging study, defined as the presence of at least one radiotracer-avid lesion considered visually typical of prostate cancer, by the consensus of three nuclear medicine physicians and one radiologist. The reported positivity rate was 801 of 1,007 patients $(80 \%)$, and multivariate analyses indicated that the likelihood of a positive finding was almost exclusively determined by PSA level: positivity rates ranged from $46 \%$ in patients with PSA $₫ 0.2 \mathrm{ng} / \mathrm{ml}$ to $57 \%$ for PSA levels $\unlhd \mathrm{ng} / \mathrm{ml}$, to $>90 \%$ in patients with $\mathrm{PSA}>3 \mathrm{ng} / \mathrm{ml}$.

To date, this study is the largest published clinical experience with any type of PSMAdirected imaging probe. Despite its retrospective design, the heterogeneous study cohort, and the lack of histopathological confirmation of positive findings, the results of this study are encouraging. The findings are in line with a 2016 meta-analysis (overall positivity rate of $81 \%, 50 \%$ in patients with PSA $0.2-0.49 \mathrm{ng} / \mathrm{ml}$ ) in the recurrent prostate cancer setting (nine studies, 983 patients), ${ }^{10}$ and compare favourably with positivity rates of ${ }^{11} \mathrm{C}$-choline and ${ }^{18}$ F-fluciclovine PET-CT reported in a recent prospective trial (for all patients: $37 \%$ and $34 \%$, respectively; for patients with PSA $<1 \mathrm{ng} / \mathrm{ml}$ : $14 \%$ and $21 \%$, respectively) ${ }^{9}$. The lack of histopathological correlation is probably the most notable drawback of this study and the true sensitivity of PSMA-PET-CT is probably lower than reported, as the list of known PSMA-expressing benign and malignant tissues expands with the increasingly widespread use of PSMA-targeting tracers. Prospective trials with histopathological correlation are under way to test the true diagnostic precision of PSMA-targeting agents.

In summary, we believe PROMIS provides evidence for the safe avoidance of TRUS biopsy in patients with a negative prostate MRI, provided that technical adequacy and qualified radiologists are available. Furthermore, PSMA-targeting PET tracers are developing into the new standard for imaging of recurrent prostate cancer, but further controlled studies are needed to fully determine their strengths and limitations.

\section{Acknowledgements}

The authors thank Ada Muellner, MS, of Memorial Sloan Kettering Cancer Center, who helped edit the manuscript before submission.

Competing interests statement

A.G.W. has received support from the Peter Michael Foundation. H.H. serves on the Board of Directors of Ion Beam Application (IBA), a role for which she receives annual compensation.

\section{References}

1. Ahmed HU et al. Diagnostic accuracy of multi-parametric MRI and TRUS biopsy in prostate cancer (PROMIS): a paired validating confirmatory study. Lancet 389, 815-822 (2017). [PubMed: 28110982]

2. Faria $\mathrm{R}$ et al. Optimising the diagnosis of prostate cancer in the era of multiparametric magnetic resonance imaging: a cost-effectiveness analysis based on the prostate MR imaging study (PROMIS). Eur. Urol 10.1016/j.eururo.2017.08.018 (2017).

3. Afshar-Oromieh A et al. Diagnostic performance of 68Ga-PSMA-11 (HBED-CC) PET/CT in patients with recurrent prostate cancer: evaluation in 1007 patients. Eur. J. Nucl. Med. Mol. Imaging 44, 1258-1268 (2017). [PubMed: 28497198] 
4. Moldovan PC et al. What is the negative predictive value of multiparametric magnetic resonance imaging in excluding prostate cancer at biopsy? A systematic review and meta-analysis from the European Association of Urology Prostate Cancer Guidelines Panel. Eur. Urol 72, 250-266 (2017). [PubMed: 28336078]

5. Delongchamps NB et al. Prebiopsy magnetic resonance imaging and prostate cancer detection: comparison of random and targeted biopsies. J. Urol 189, 493-499 (2013). [PubMed: 22982424]

6. Puech P et al. Prostate cancer diagnosis: multiparametric MR-targeted biopsy with cognitive and transrectal US-MR fusion guidance versus systematic biopsy - prospective multicenter study. Radiology 268, 461-469 (2013). [PubMed: 23579051]

7. Gillessen S et al. Management of patients with advanced prostate cancer: the report of the advanced prostate cancer consensus conference APCCC 2017. Eur. Urol 10.1016/j.eururo.2017.06.002 (2017).

8. Vargas HA et al. Localizing sites of disease in patients with rising serum prostate-specific antigen up to $1 \mathrm{ng} / \mathrm{ml}$ following prostatectomy: how much information can conventional imaging provide? Urol. Oncol 34, 482.e5-482.e10 (2016).

9. Nanni C et al. 18F-FACBC (anti1-amino-3-18F-fluorocyclobutane-1-carboxylic acid) versus 11Ccholine PET/CT in prostate cancer relapse: results of a prospective trial. Eur. J. Nucl. Med. Mol. Imaging 43, 1601-1610 (2016). [PubMed: 26960562]

10. von Eyben FE et al. 68Ga-labeled prostate-specific membrane antigen ligand positron emission tomography/computed tomography for prostate cancer: a systematic review and meta-analysis. Eur. Urol. Focus 10.1016/j.euf.2016.11.002 (2016). 


\section{Key advances}

- $\quad$ PROMIS provides level 1 evidence that prostate MRI has a higher sensitivity and lower specificity for the diagnosis of prostate cancer than transrectal ultrasonography-guided (TRUS) biopsy ${ }^{1}$

- $\quad$ The results of PROMIS substantiate the feasibility and safety of avoiding TRUS biopsy in patients with a negative MRI ${ }^{1}$

- $\quad$ Prostate MRI followed by targeted TRUS biopsy of suspicious lesions is a cost-effective strategy for prostate cancer diagnosis ${ }^{2}$

- $\quad$ PSMA-targeting PET tracers seem to provide unique sensitivity for the detection and localization of recurrent and metastatic prostate cancer ${ }^{3}$ 